\title{
Post-surgical Gangrene with Pseudomonas luteola Resulting in Limb Amputation: A Case Review
}

\author{
Wallisa Roberts ${ }^{1}$, Carmen Roessler ${ }^{2}$, Petra J. Francis ${ }^{3}$, Dolland Noel ${ }^{4}$, Marios Loukas ${ }^{5}$ \\ 1. General Surgery, Grenada General Hospital, St. George, GRD 2. Anatomy, St. George's University, St. George, GRD 3. \\ Surgery, Grenada General Hospital, St. George, GRD 4. Internal Medicine, Grenada General Hospital, St. Georges, GRD \\ 5. Anatomy, St. George's University, St. George's, GRD
}

Corresponding author: Marios Loukas, mloukas@sgu.edu

\section{Abstract}

Pseudomonas luteola is a rare infective agent with a variable resistance-sensitivity panel. Clinical suspicion and appropriate empiric treatment is necessary for resolution of such infections. We report a case of postsurgical gangrene as a result of Pseudomonas luteola culminating in limb amputation.

Categories: General Surgery, Infectious Disease

Keywords: pseudomonas, amputation, gangrene, luteola

\section{Introduction}

Pseudomonas luteola (P. luteola) is an aerobic, motile Gram-negative rod appearing as light to deep shades of yellow colonies on MacConkey or blood agar. However, it differs from most of the other members of the Pseudomonas group, as it is oxidase negative [1]. P. luteola has clinical significance as it has been implicated in a variety of life-threatening infections such as: endocarditis, peritonitis, meningitis, septicemia and brain abscesses [2-4]. Moreover, it has also been diagnosed as the causal factor in less common infections such as: endophthalmitis, mediastinal botryomycosis and osteomyelitis [5-7].

P. luteola was initially designated to the CDC biogroup Ve-1 group due to its multi-trichous polar flagella [8]. The bacteria underwent several name and genus changes: Chromobacterium typhiflavum, Chryseomonas polytrichia, and Chryseomonas luteola, until 1997, when Anzai et al. performed a 16S rRNA sequence analysis which revealed the synonymy of the Chryseomonas, Flavimonas and Pseudomonas groups [9]. We report a case of $P$. luteola isolation from a traumatic wound that later resulted in lower limb amputation.

Received 08/20/2018

Review began 10/02/2018 Review ended 10/09/2018 Published 10/11/2018

๑) Copyright 2018

Roberts et al. This is an open access article distributed under the terms of the Creative Commons Attribution License CC-BY 3.0., which permits unrestricted use, distribution, and reproduction in any medium, provided the original author and source are credited.

\section{Case Presentation}

A 43-year-old man was brought to the emergency room with deep machete lacerations to the right forearm, a superficial laceration to the left forearm, and a deep laceration and partial amputation of the right lower leg (Figure 1). 


\section{Cureus}

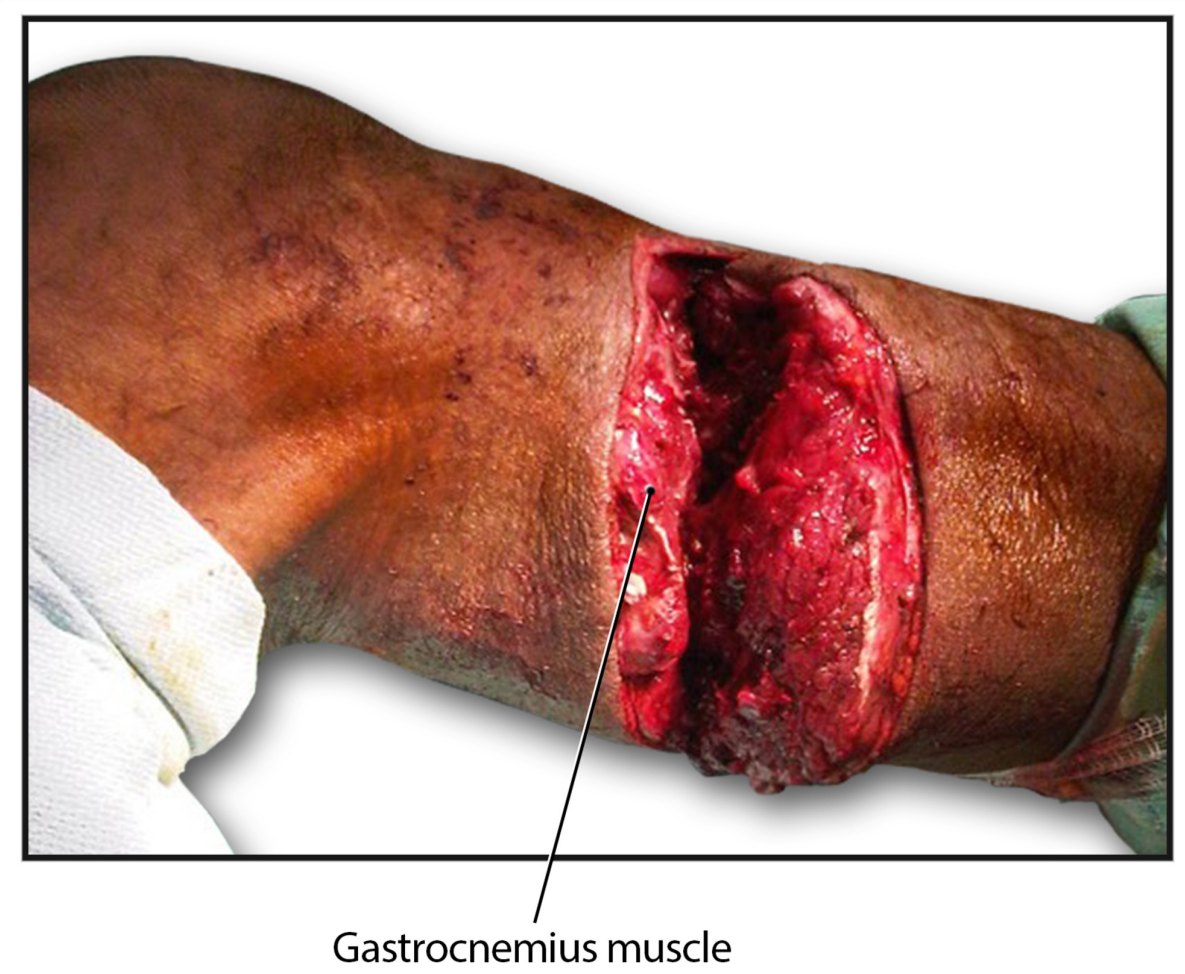

FIGURE 1: Partial amputation of the patient's right leg secondary to a traumatic injury.

Radiographs showed complete comminuted and displaced fractures of the right midshaft of the radius and ulna, as well as a comminuted fracture of the left proximal fibula (Figures 2).
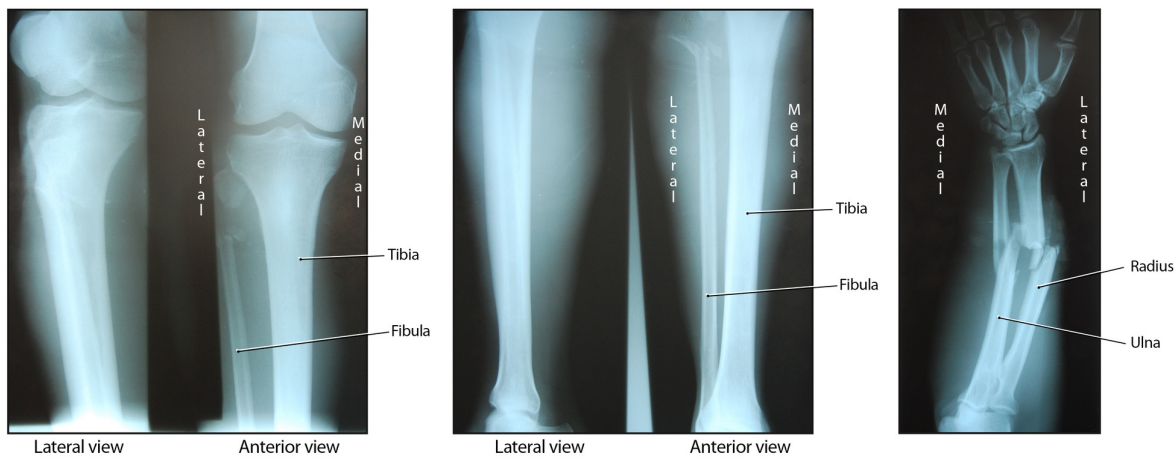

FIGURE 2: Radiographic images of the patient's polytraumatic presentation.

A chest radiograph was unremarkable. His past medical history included asthma and a hospital admission for an abscess of the right thumb in 2009.

Immediate care included antibiotic coverage with cloxacillin $500 \mathrm{mg}$ IV every six hours and gentamicin 80 $\mathrm{mg}$ IV every eight hours. Intraoperative reconstruction and external fixation of the forearm was performed, as well as reconstruction of the fibula with common peroneal nerve and gastrocnemius muscle repair. Postoperatively, cloxacillin and gentamicin were continued for a total of seven and five days, respectively.

Five days after admission, the patient developed signs of infection in the right lower limb and debridement was performed twice in the operating theatre. Ceftazidime $1 \mathrm{~g}$ IV every eight hours and crystapen

benzylpenicillin two megaunits IV every six hours were added to the antibiotic regime. Wound swabs were obtained, and Gram stains showed numerous Gram-negative bacilli and some Gram-positive cocci, as well as 


\section{Cureus}

yeast cells. However, wound cultures grew only $P$. aeruginos a and $P$. luteola. The $P$. aeruginosa was sensitive to amikacin, ceftazidime, ciprofloxacin, gentamicin and tobramycin, but was resistant to carbenicillin. $P$. luteola displayed sensitivity to amikacin, ceftazidime, ciprofloxacin, gentamicin and tobramycin with resistance to ampicillin, augmentin, bactrim and imipenem, and intermediate sensitivity to cefotaxime and ceftriaxone.

On hospital day seven, the patient spiked a temperature of $38.1^{\circ} \mathrm{C}$ and the lower limb showed signs of gangrene (Figure 3); the foot was cold and swollen with loss of sensation, peripheral pulses were absent, and palpable crepitations were present. A diagnosis of gas gangrene was made.

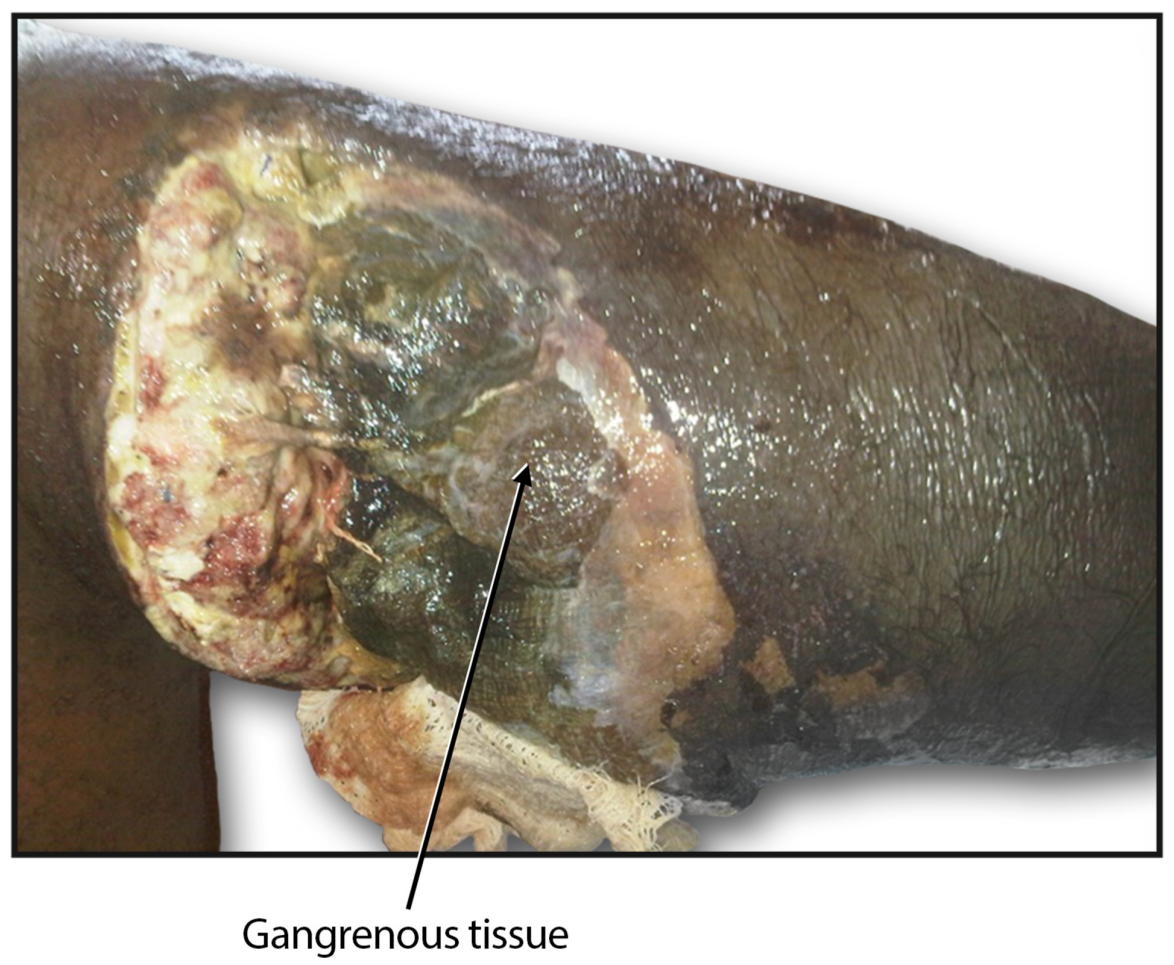

FIGURE 3: Postoperative complication of gangrene of the right leg.

The patient and his family were informed of the available treatment options and together with the medical team, a decision was made to perform an above-knee amputation of the right leg. Post amputation, the patient's health improved, and the rest of his hospital stay was uneventful. Three months after the trauma incident, the right stump was clean and healing well. The fractures of the right forearm were also healing, and good callus formation was seen.

\section{Discussion}

In the Caribbean and particularly in Grenada, diabetes is usually the leading cause of lower limb amputations [10]. Although Staphylococcus aureus is the organism most commonly associated with such infections leading to lower extremity amputations, the Pseudomonas genus is also known to cause gangrene resulting in amputation [11]. P. luteola has been isolated from both ulcers and osteomyelitis cases, but as far as we are aware, no such cases have resulted in amputation of a limb $[6,7,12]$. To the best of our knowledge, this is the first reported case of $P$. luteola infection in Grenada, and quite possibly the first case of $P$. luteola to be isolated from a wound that later resulted in amputation.

P. luteola is an infrequent human pathogen. The natural habitat of the pathogen has not been determined, but it belongs to a group of bacteria that are usually found in water, soil, and other moist environments [13]. In most clinical cases to date, it has been associated with foreign bodies and indwelling catheters often mandating removal [1], corticosteroid use, and other immuno-compromised states [1, 7,14,15]. In both of the previous reports of $P$. luteola-induced osteomyelitis, patients were healthy with no known underlying medical conditions, and with no reasons to be chronically immuno-compromised. In this case, the patient was a healthy individual, who had not used any steroids, and no long-standing extraneous matter was found to be present in the leg.

As found in our circumstance, in vitro studies have typically demonstrated the susceptibility of $P$. luteola to 


\section{Cureus}

aminoglycosides and fluoroquinolones such as ciprofloxacin. However, variable resistance has been noted against many $\beta$-lactam-containing antibiotics such as penicillins and cephalosporins [12]. This prompted the discovery of blaLUT-1 and other new $\beta$-lactamase gene variants by Doublet et al. [13]. It is important to acknowledge that due to its variable resistance pattern, this bacterium should be considered as a possible culprit when infections are un-resolving - particularly in immuno-compromised patients and those with indwelling foreign bodies $[1,13]$. As was seen in this instance, $P$. luteola can be involved in infections that lead to severe morbidity, and may even result in mortality. In their retrospective study, Bayhan et al. assessed the number of cases of $P$. luteola at a pediatric hospital [16]. They found that the majority of cases of $P$. luteola ( $85.7 \%, 6 / 7$ patients) were hospital acquired and that nosocomial infection by the pathogen tended to present as bacteremia. From their sensitivity-resistance panel, they suggested that carbapenems were the most appropriate agent of choice for nosocomial $P$. luteola as all their isolates were susceptible to this class of drug, even though currently no standardized regimen exists. P. luteola has a relapsing/chronic course when the right antibiotic treatment is not selected [7]. Therefore, the authors feel a consensus regarding imperative therapy is needed. Thus, from our preliminary review of the sensitivity and resistance reported in the case studies $[2,3,7,12,14-19]$ examined (Table 1 ), the authors would like to suggest that a suitable initial therapy when this organism is encountered might be a beta-lactam and aminoglycoside, specifically imipenem and amikacin.

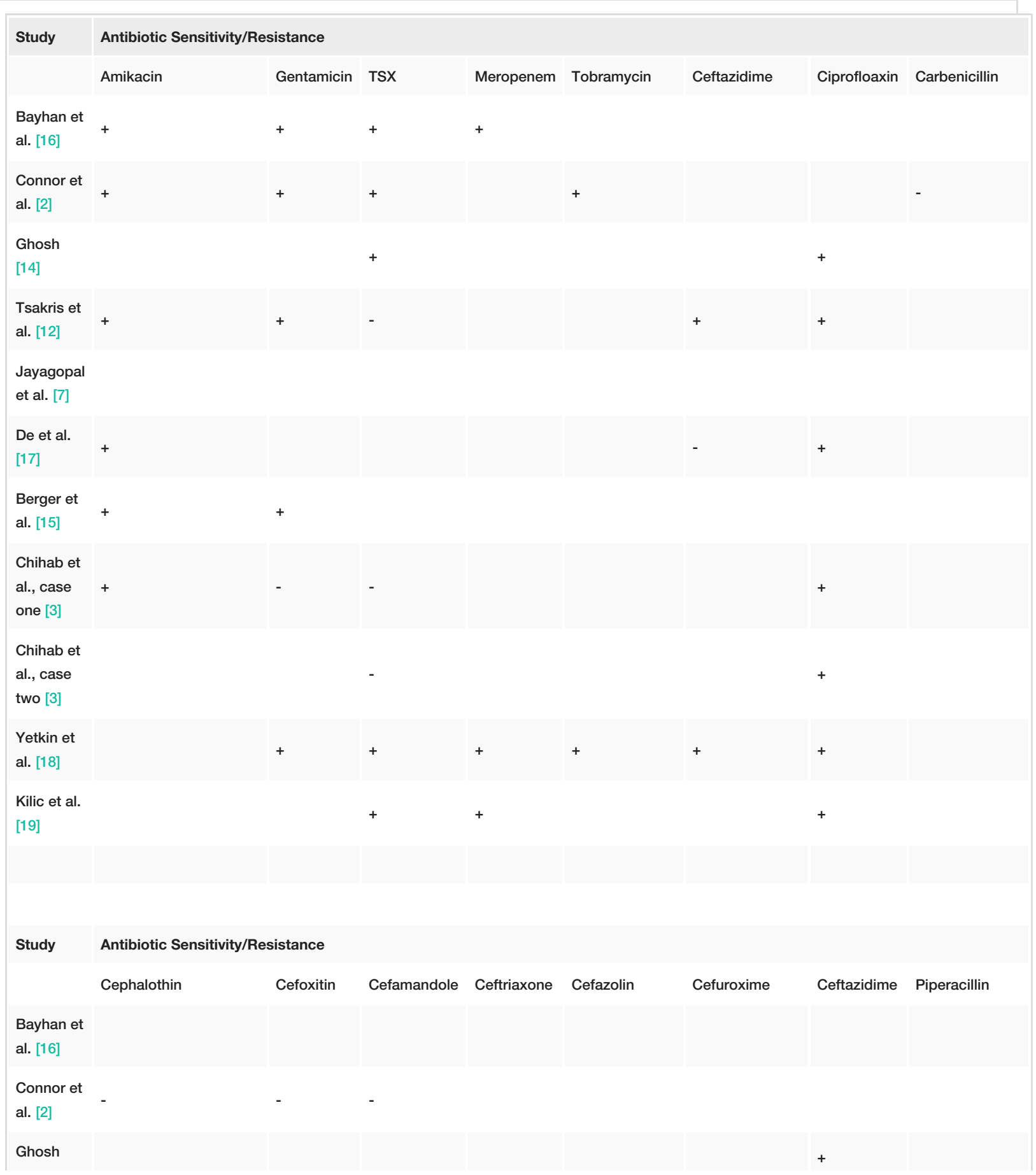




\section{Cureus}

[14]

Tsakris et

al. [12]

Jayagopal

et al. [7]

De et al.

[17]

Berger et

al. [15]

Chihab et

al., case

one [3]

Chihab et

al., case

two [3]

Yetkin et

al. [18]

Kilic et al.

[19]

$\sqrt{2}$

Study Antibiotic Sensitivity/Resistance

Piperacillin/tazobactam Cefepime Imipenem Pefloxacin Tobramycin Ampicillin Amoxicillin Amoxiclav

Bayhan et

al. [16]

Connor et

al. [2]

Ghosh

[14]

Tsakris et

al. [12]

Jayagopal

et al. [7]

De et al.

[17]

Berger et

al. [15]

Chihab et

al., case

one [3]

Chihab et

al., case

two [3]

Yetkin et

al. [18]

Kilic et al.

[19] 


\section{Cureus}

Study

Antibiotic Sensitivity/Resistance

$\begin{array}{llll}\text { Cephalothin } & \text { Cefoxitin Norfloaxin } & \begin{array}{l}\text { Nalidixic } \\ \text { acid }\end{array}\end{array}$ Oxytetracycline Colistin

Bayhan et

al. [16]

Connor et

al. [2]

Ghosh

[14]

Tsakris et

al. [12]

Jayagopal

et al. [7]

De et al.

[17]

Berger et

al. [15]

Chihab et

al., case

one [3]

Chihab et

al., case

two [3]

Yetkin et

al. [18]

Kilic et al.

[19]

Study Antibiotic Sensitivity/Resistance

Ofloxacin Cefotaxime Doxycycline Netilmicin Ureidopenicillin Fluoroquinolone Tetracycline Cefoperasone

Bayhan et

al. [16]

Connor et

al. [2]

Ghosh

[14]

Tsakris et

al. [12]

Jayagopal

et al. [7]

De et al.

[17]

Berger et

al. [15]

Chihab et

al., case

one [3]

Chihab et 


\section{Cureus}

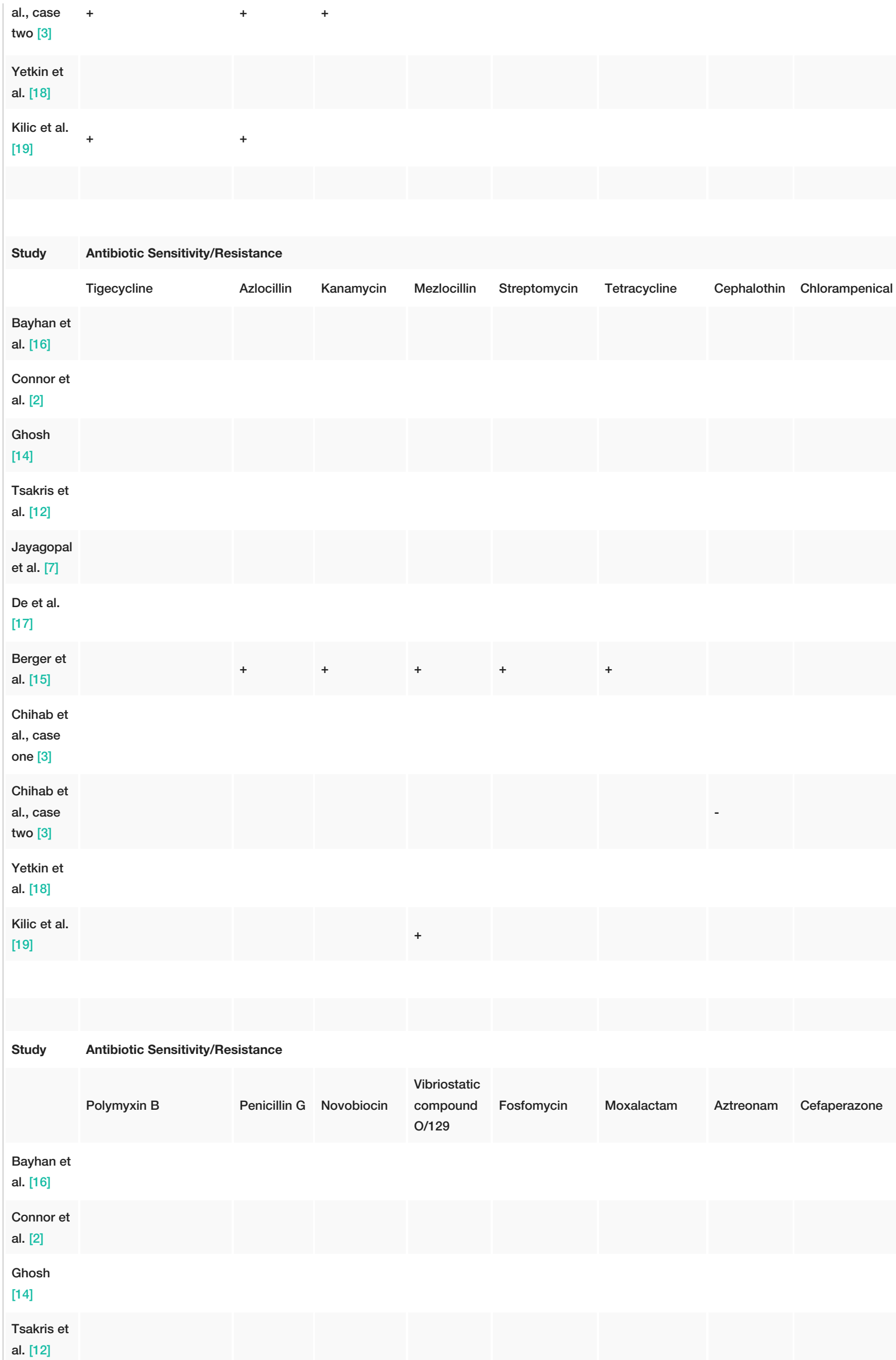

Polymyxin B Penicillin G Novobiocin compound Fosfomycin $\quad$ Moxalactam Aztreonam Cefaperazone

Bayhan et

al. [16]

Connor et

al. [2]

Ghosh

[14]

Tsakris et

al. [12] 


\section{Cureus}

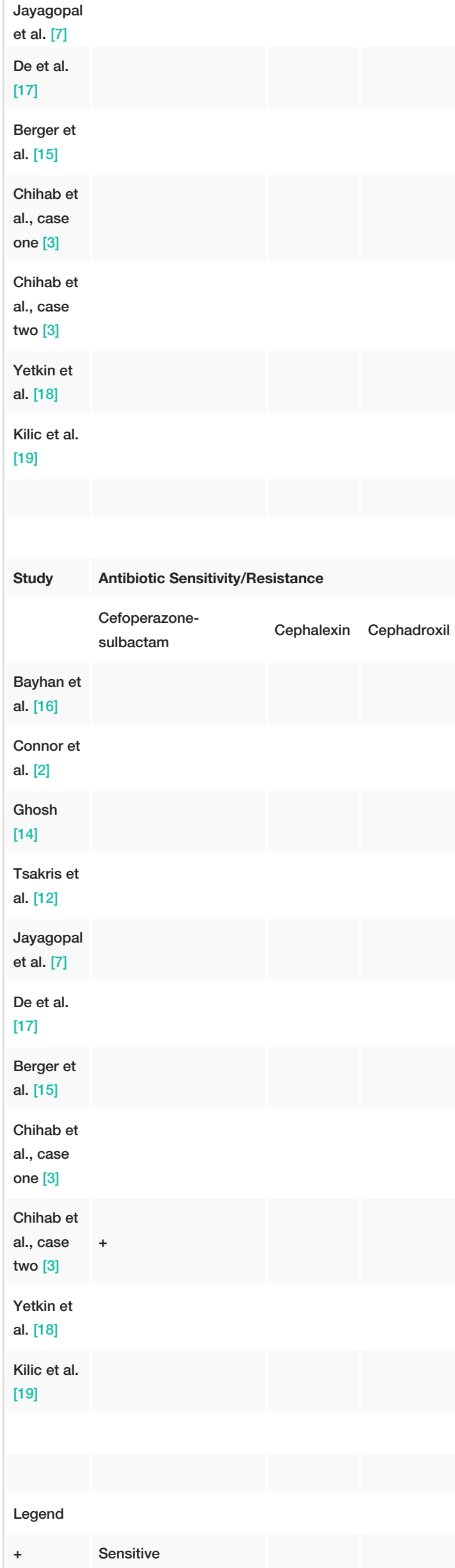




\section{Cureus}

Resistant

Unavailable

TABLE 1: Preliminary overview of the resistance and sensitivity panel of P. luteola based on case reports.

This drug combination was also chosen due to the fact that $P$. luteola tends to present with septicemia [5]. Furthermore, Imipenem was chosen as an appropriate empiric drug, despite our isolate resistance to it, as generally from the preliminary panel, $P$. luteola tends to be sensitive to Imipenem. Tamma et al., in their study, showed that for severely ill patients secondary to gram-negative infections, combination therapy resulted in better survivability than monotherapy in certain subgroups [20].

\section{Conclusions}

In summary, due to its serious clinical implications, as well as the fact that it has been identified as a nosocomial infection, it is imperative that microbiologists and physicians alike are aware of $P$. luteola, particularly due to its multidrug resistance variability, and the chronic/relapsing course that is seen when the organism is not treated with the correct antibiotics. We therefore suggest a beta-lactam/aminoglycoside combination of amikacin/imipenem. We recognize that further research is needed to elucidate a definitive imperative treatment.

\section{Additional Information \\ Disclosures}

Human subjects: Consent was obtained by all participants in this study. Conflicts of interest: In compliance with the ICMJE uniform disclosure form, all authors declare the following: Payment/services info: All authors have declared that no financial support was received from any organization for the submitted work. Financial relationships: All authors have declared that they have no financial relationships at present or within the previous three years with any organizations that might have an interest in the submitted work. Other relationships: All authors have declared that there are no other relationships or activities that could appear to have influenced the submitted work.

\section{References}

1. Otto MP, Foucher B, Dardare E, Gerome P: Severe catheter related bacteremia due to Pseudomonas luteola . Med Mal Infect. 2013, 43:170-171. 10.1016/j.medmal.2013.01.013

2. Connor BJ, Kopecky RT, Frymoyer PA, Forbes BA: Recurrent Pseudomonas luteola (CDC group Ve-1) peritonitis in a patient undergoing continuous ambulatory peritoneal dialysis. J Clin Microbiol. 1987, 25:1113-1114.

3. Chihab W, Alaoui AS, Amar M: Chryseomonas luteola identified as the source of serious infections in a Moroccan university hospital. J Clin Microbiol. 2004, 42:1837-1839. 10.1128/JCM.42.4.1837-1839.2004

4. Gaschet A, Engrand C, Piau C, et al.: Multiple brain abscesses caused by Pseudomonas luteola . Pediatr Infect Dis J. 2009, 28:1144-1146. 10.1097/INF.0b013e3181bd8158

5. Goteri G, Dorrestein GM, Manso E, Giantomassi F, Rubini C, Zizzi A, Pomponio G: Chryseomonas luteola: an unusual clinical infection mimicking a mediastinal malignant lymphoma. Pathol Lab Med Int. 2010, 2:137139. 10.2147/PLMI.S13645

6. Rahav G, Simhon A, Mattan Y, Moses AE, Sacks T: Infections with Chryseomonas luteola (CDC group Ve-1) and flavimonas oryzhabitans (CDC group Ve-2). Medicine (Baltimore). 1995, 74:83-88.

7. Jayagopal S, Berry MG, Ross G, Howcroft AJ: Hand infection caused by Chryseomonas luteola. Br J Plast Surg. 2004, 57:694-695. 10.1016/j.bjps.2004.05.028

8. Holmes B, Steigerwalt AG, Weaver RE, Brenner DJ: Chryseomonas luteola comb. nov. and Flavimonas oryzihabitans gen. nov., comb. nov., Pseudomonas-like species from human clinical specimens and formerly known, respectively, as Groups Ve-1 and Ve-2. Int J Syst Evol Microbiol. 1987, 37:245-250. 10.1099/00207713-37-3-245

9. Anzai Y, Kudo Y, Oyaizu H: The phylogeny of the genera Chryseomonas, Flavimonas, and Pseudomonas supports synonymy of these three genera. Int J Syst Evol Microbiol. 1997, 47:249-251. 10.1099/0020771347-2-249

10. Hambleton IR, Jonnalagadda R, Davis CR, Fraser HS, Chaturvedi N, Hennis AJ: All-cause mortality after diabetes-related amputation in Barbados: a prospective case-control study. Diabetes Care. 2009, 32:306-307. 10.2337/dc08-1504

11. Dunn DL: Diagnosis and treatment of infection. Surgery: Basic Science and Clinical Evidence. Norton JA, Barie PS, Bollinger RR, et al. (ed): Springer, New York; 2008. 209-235.

12. Tsakris A, Hassapopoulou H, Skoura L, Pournaras S, Doubouyas J: Leg ulcer due to Pseudomonas luteola in a patient with sickle cell disease. Diagn Microbiol Infect Dis. 2002, 42:141-143. 10.1016/S07328893(01)00336-4

13. Doublet B, Robin F, Casin I, Fabre L, Le Fleche A, Bonnet R, Weill FX: Molecular and biochemical characterization of the natural chromosome-encoded Class A Beta-lactamase from Pseudomonas luteola. 


\section{Cureus}

Antimicrob Agents Chemother. 2010, 54:45-51. 10.1128/AAC.00427-09

14. Ghosh SK: A rare infection caused by Chryseomonas luteola . J Infect. 2000, 41:109-110.

10.1053/jinf.2000.0660

15. Berger SA, Siegman-Igra Y, Stadler J, Campus A: Group Ve-1 septicaemia. J Clin Microbiol. 1983, 17:926-927.

16. Bayhan GI, Senel S, Tanir G, Ozkan S: Bacteremia caused by Pseudomonas luteola in pediatric patients . Ipn J Infect Dis. 2015, 68:50-54. 10.7883/yoken.JJID.2014.051

17. De AS, Salunke PP, Parikh HR, Baveja SM: Chryseomonas luteola from bile culture in an adult male with severe jaundice. J Lab Physicians. 2010, 2:40-41. 10.4103/0974-2727.66708

18. Yetkin G, Bayraktar MR, Sivri N, Kosar F: An unusual infection of Chryseomonas luteola: infective endocarditis in a patient with ventricular septal defect. Ann Med Res. 2005, 12:193-195.

19. Kilic A, Baysallar M, Yildiz C, Kucukkaraaslan A, Doganci L, Widmann R: A rare case of septic arthritis of shoulder joint caused by Chryseomonas luteola. Anatol J Clin Investing. 2008, 2:43-44.

20. Tamma PD, Cosgrove SE, Maragakis LL: Combination therapy for treatment of infections with gramnegative bacteria. Clin Microbiol Rev. 2012, 25:450-470. 10.1128/CMR.05041-11 